\title{
ANÁLISE DA HIDRODINÂMICA DE UM CIRCUITO INDUSTRIAL DE CIANETAÇÃO DE OURO
}

\author{
E. B. JESUS, C. S. A. VASCONCELOS e L. R. P.de ANDRADE LIMA* \\ Departamento de Ciência e Tecnologia dos Materiais - Universidade Federal da Bahia \\ lelo@ufba.br*
}

Artigo submetido em novembro/2013 e aceito em novembro/2014

DOI: $10.15628 /$ holos.2014.1830

\section{RESUMO}

A lixiviação por cianeto em meio alcalino tem sido o principal método utilizado para a extração de ouro de minérios. Esse processo é predominantemente realizado em tanques agitados que são típicos reatores heterogêneos não-catalíticos onde ocorrem múltiplas reações envolvendo as fases gasosa, líquida e sólida. Por esta razão, a qualidade da mistura das fases no interior dos tanques é fundamental para a otimização deste processo. Neste trabalho foi avaliado a hidrodinâmica de um circuito de lixiviação industrial a partir da interpretação dos resultados obtidos de um ensaio com traçador. Um teste com um traçador foi utilizado para avaliar as características de um circuito de cianetação com nove reatores. Amostras foram coletadas na saída de quatro tanques. As distribuições de tempos de residência foram determinadas e analisadas. Arranjos de reatores ideais foram utilizados para modelar as distribuições e os parâmetros foram ajustados aos dados experimentais através do método dos mínimos quadrados. Os tempos médios de residência foram sempre menores que os tempos nominais de residência, o que sugere a redução do volume útil dos tanques. As análises das distribuições indicam a presença de discretas zonas de estagnação ao longo da cascata. A modelagem dos resultados sugere que $63 \%$ dos volumes dos tanques podem ser explicados pela associação de reatores de mistura perfeita.

PALAVRAS-CHAVE: Lixiviação, Ouro, Circuito, Distribuição de tempos de residência

\section{ANALYSIS OF THE HYDRODYNAMIC BEHAVIOR OF AN INDUSTRIAL GOLD CYANIDATION CIRCUIT}

\begin{abstract}
The cyanide leaching under alkaline conditions has been the primary method used for extraction of gold from ores. This process is mainly carried out in stirred tanks which are typical non-catalytic reactors where there heterogeneous multiple reactions involving the gaseous, liquid and solid phases. For this reason, the quality of mixing of the phases within the tanks is essential to optimize this process. This study evaluated the hydrodynamics of an industrial leaching circuit from the interpretation of the results of a tracer test. A tracer experiment was used to evaluate the characteristics of a gold cyanidation circuit with nine tanks. Samples were
\end{abstract}

collected at the exit four tanks of the cascade. The residence time distributions were determined and analyzed. Arrangements of ideal reactors were used to fit the distributions using the least squares method. The average residence times were always lower than the nominal residence time, suggesting the reduction of the total volume of the tanks. The analysis of the distributions indicates the presence of small stagnant zones along the cascade. The mathematical modeling of the results suggests that about $63 \%$ of the volumes of the tanks can be explained by the association of continuous stirredtank reactor.

KEYWORDS: Leaching, Gold, Circuit, Residence time distribution 


\section{INTRODUÇÃO}

A lixiviação por cianeto em meio alcalino tem sido o principal método utilizado para a extração de ouro de minérios por mais de um século. Esse processo é predominantemente realizado em tanques agitados mecanicamente ou através de injeção de ar (HABASHI, 1987). Os tanques de lixiviação são típicos reatores heterogêneos não-catalíticos onde ocorrem múltiplas reações envolvendo as fases gasosa, líquida e sólida. Em função da grande quantidade de material processado e da pequena velocidade da reação de cianetação do ouro, os tanques de lixiviação utilizados nas plantas industriais geralmente possuem grandes volumes, normalmente entre 100 e $1500 \mathrm{~m}^{3}$ (STEGOWSKI et al., 2010). Embora, os tanques sejam acoplados e possuam mecanismos de agitação de polpa, estes podem exibir zonas de estagnação e formação de caminhos preferenciais, os quais podem reduzir o desempenho do processo (DE ANDRADE LIMA e HODOUIN, 2005; STEGOWSKI et al., 2010). O comportamento hidrodinâmico dos tanques e dos circuitos industriais de lixiviação não é bem conhecido, embora seja de grande importância para a compreensão e para a otimização dos processos industriais de extração de ouro (DE ANDRADE LIMA e HODOUIN, 2005).

A análise da distribuição dos tempos de residência (DTR) é uma clássica ferramenta utilizada para avaliar o desempenho de reatores químicos não-ideais e circuitos industriais (LELINSKI et al., 2002; NEWCOMBE et al., 2013) a exemplo do diagnóstico de zonas de estagnação e formação de caminhos preferenciais, assim como da existência de dispersão axial e zonas mortas (DANCKWERTS, 1953; LEVENSPIEL, 1999). Essa analise tem sido utilizada para desenvolver modelos realísticos e descrever os complexos reatores e circuitos industriais (LEVENSPIEL, 1999; NAUMAN e BUFFHAM, 1983). Os experimentos para a obtenção da DTR consistem em ensaios de estímuloresposta (NAUMAN e BUFFHAM, 1983) e as formas mais comuns de estímulo são: impulso, degrau positivo ou negativo, rampa, senóide ou variações aleatórias.

\section{ASPECTOS TEÓRICOS}

$\mathrm{Na}$ abordagem do balanço populacional, o padrão de fluxo e as propriedades de mistura dos vários elementos de fluidos, moléculas ou partículas nos reatores são caracterizados por duas funções: a distribuição da idade interna (I) e a distribuição dos tempos de residência $(E)$, que estão relacionadas (HIMMELBLAU e BISCHOFF, 1968):

$$
E(t)=-\frac{d I(t)}{d t}
$$

A distribuição dos tempos de residência dos reatores é usualmente determinada com o uso de traçadores, que devem ser relativamente inertes e apresenta comportamento análogo aos elementos de fluido a serem caracterizados. Em processos minero-metalúrgicos a perturbação geralmente é feita na forma de pulso ou degrau. Assumindo que a vazão é relativamente constante, a DTR pode ser estimada através da normalização das concentrações do traçador na saída do reator (C) (HIMMELBLAU e BISCHOFF, 1968): 


$$
E(t)=\frac{C(t)}{\int_{0}^{\infty} C(t) d t}
$$

Nota-se que:

$\int_{0}^{\infty} E(t) d t=1$

O tempo médio de residência do reator $(\tau)$ pode ser estimado por (HIMMELBLAU e BISCHOFF, 1968):

$$
\tau=\frac{\int_{0}^{\infty} t C(t) d t}{\int_{0}^{\infty} C(t) d t}
$$

As funções de distribuição de idade podem ser expressas em função do tempo adimensional $(\theta=t / \tau)$ como segue (HIMMELBLAU e BISCHOFF, 1968):

$$
\begin{aligned}
& E(\theta)=\tau E(t) \\
& I(\theta)=\tau I(t)
\end{aligned}
$$

Para modelar as curvas de DTR modelos fenomenológicos, baseados nas equações de balanço de massa, quantidade de movimento e energia, podem ser usados, entretanto o uso de uma combinação de reatores ideais simples, como misturadores perfeitos (CSTR) e reatores de fluxo pistonado (PRF) pode descrever muitos casos reais e representam um esforço matemático reduzido (BAZIN e HODOUIN, 1988). A arquitetura do arranjo de reatores deve ser selecionada de tal modo a exigir o menor número de parâmetros possível, enquanto ajusta o formato da curva experimental da DTR. As configurações dos reatores e os parâmetros dos modelos podem ser estimados utilizando o critério dos mínimos quadrados que por sua vez pode ser minimizado, com respeito aos parâmetros do modelo (DE ANDRADE LIMA e HODOUIN, 2005).

O objetivo deste trabalho é avaliar o comportamento hidrodinâmico de um circuito de lixiviação industrial a partir da interpretação dos resultados obtidos de um ensaio com traçador. Os resultados apresentados correspondem ao comportamento da fase líquida da polpa. Embora exista diferença de comportamento entre a fase líquida, o minério e o ouro, a segregação das partículas nesta parte do circuito é muito menor que em outros setores da planta de beneficiamento, como é o caso do circuito fechado de moagem-classificação, que segrega as partículas mais densas resultando em maior tempo de retenção no circuito.

\section{MATERIAIS E MÉTODOS}

Os experimentos foram realizados na planta de beneficiamento da Mineração Fazenda Brasileiro, localizada no município de Barrocas, Bahia. Na Figura 1, é apresentado um dos circuitos 
de lixiviação do minério aurífero utilizado pela planta hidrometalúrgica que processa industrialmente minério de ouro. Inicialmente a polpa é condicionada com a adição de hidróxido de cálcio nos tanques 01 e 03, para ajustar o pH em 12. Nos tanques 01, 02 e 03 ar comprimido é injetado, na parte inferior do reator, para promover a aeração. A agitação mecânica é utilizada a partir do tanque 03 e no tanque 04 é adicionado o cianeto de sódio diluído para solubilizar o ouro. A polpa é transferida entre os tanques por diferença de gravidade. A recuperação do ouro da fase líquida é realizada por adsorção em carvão ativado, que é adicionado ao processo no tanque 24 , e vai sendo transferido por bombeamento da polpa em contracorrente com fluxo normal da polpa até o tanque 05 onde o carvão enriquecido é retirado. A capacidade volumétrica nominal individual dos seis primeiros tanques é de $350 \mathrm{~m}^{3}$ e de $112 \mathrm{~m}^{3}$ para os demais tanques da cascata.

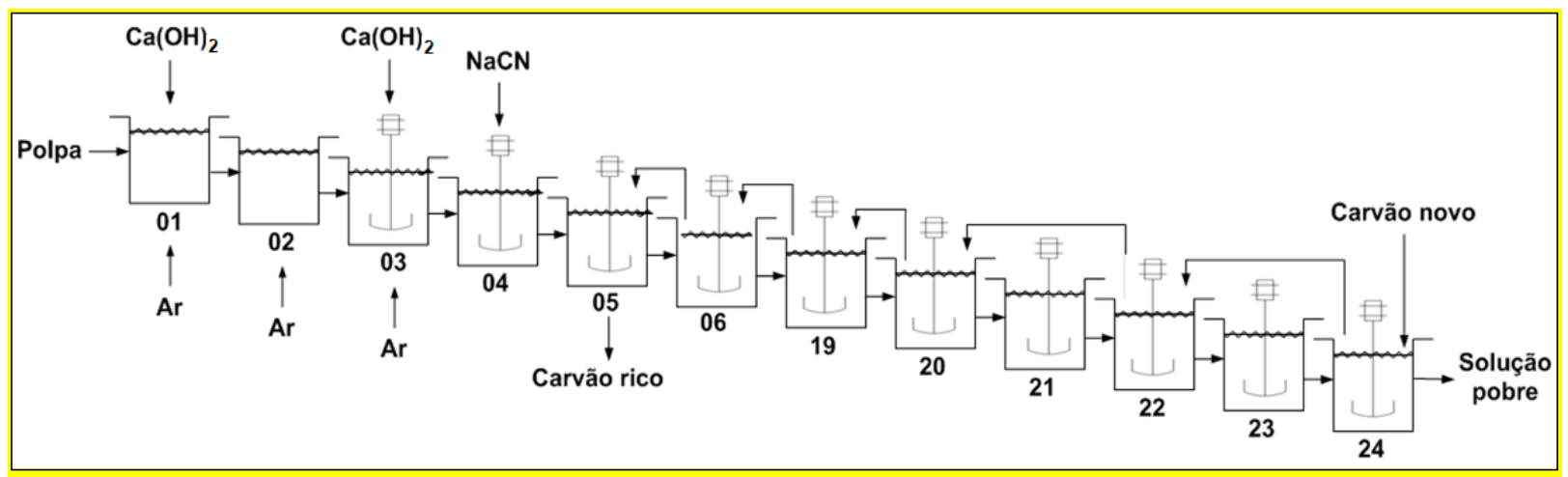

Figura 1 - Circuito de lixiviação industrial de minério aurífero - linha 1

A caracterização hidrodinâmica do circuito de lixiviação foi obtida através da realização do ensaio de estímulo-resposta com cloreto de lítio, pois o lítio não ocorre em concentração significativa na polpa, pode ser facilmente analisado na fase líquida e usualmente este elemento não interage de maneira significativa com o minério ou a solução (DE ANDRADE LIMA e HODOUIN, 2005). No presente caso o ensaio consistiu na injeção, na forma de pulso, de $20 \mathrm{~kg} \mathrm{de} \mathrm{LiCl}$ dissolvidos em 50 litros de água no primeiro tanque da cascata de lixiviação (tanque 04) enquanto as amostras foram coletadas nas saídas dos tanques 04, 05, 06 e 24 (vide Figura 1). Os tempos de coleta foram determinados previamente após simulações do circuito realizadas com os dados industriais da planta.

Durante o ensaio, o fluxo de materiais foi mantido aproximadamente constante com vazão média de sólidos de 71,25 t/h. O circuito de lixiviação foi operado sem a mobilização do carvão de modo que nenhum reciclo foi executado durante o ensaio. As amostras foram coletadas em recipientes de $250 \mathrm{~mL}$ e vedadas em seguida. No laboratório, as amostras foram filtradas e a fase líquida foi utilizada para a determinação da concentração de lítio. As análises foram feitas por espectrofotometria de absorção atômica.

\section{RESULTADO E DISCUSSÕES}

Durante o experimento, a vazão média de sólidos no circuito da linha 1 foi de 37,05 t/h enquanto a percentagem de sólidos na polpa foi mantida em torno de $52 \%$. A vazão de líquido $\left(Q_{L}\right)$, a vazão de polpa $\left(Q_{P}\right)$, a densidade da polpa $\left(\rho_{P}\right)$ e o tempo nominal de residência da polpa $\left(\tau_{n}\right)$ nos circuitos podem ser calculados através das equações: 


$$
\begin{aligned}
& Q_{L}=\left(\frac{100}{C w}-1\right) Q_{S} \\
& Q_{P}=Q_{L}+Q_{S} \\
& \tau=\frac{V \rho_{P}}{Q_{P}} \\
& \rho_{P}=\frac{1}{\frac{1}{\rho_{S}} C w+\frac{1}{\rho_{L}}(1-C w)}
\end{aligned}
$$

onde, $\rho_{S}$ e $\rho_{L}$ são as massas especificas do minério e da água, respectivamente; $V$ é o volume do equipamento ou circuito; $C w$ é a fração mássica de sólidos na polpa e $Q_{s}$ é a vazão mássica de sólidos. Durante o ensaio, a vazão média de líquido foi $34,20 \mathrm{t} / \mathrm{h}$ e a vazão média da polpa foi e $71,25 \mathrm{t} / \mathrm{h}$, enquanto que a densidade média da polpa foi $1,51 \mathrm{t} / \mathrm{m}^{3}$.

$$
\tau=0,101 \tau_{n}^{1,613}
$$

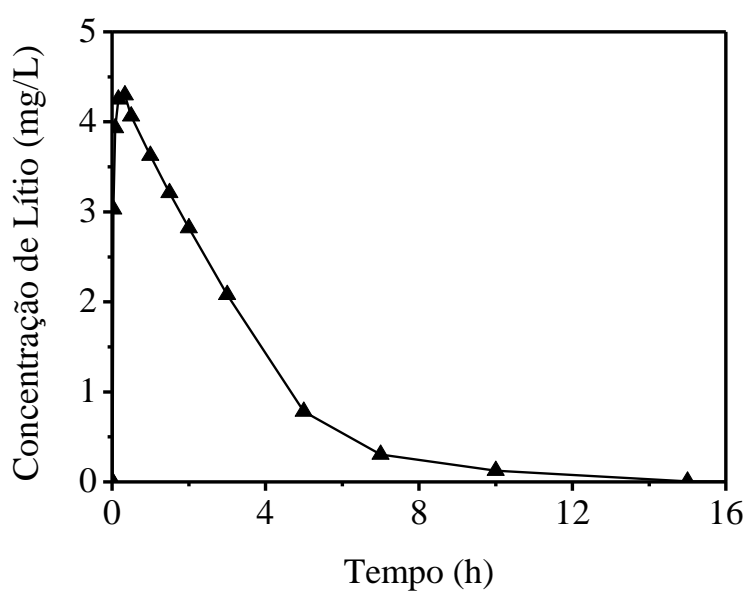

(a)

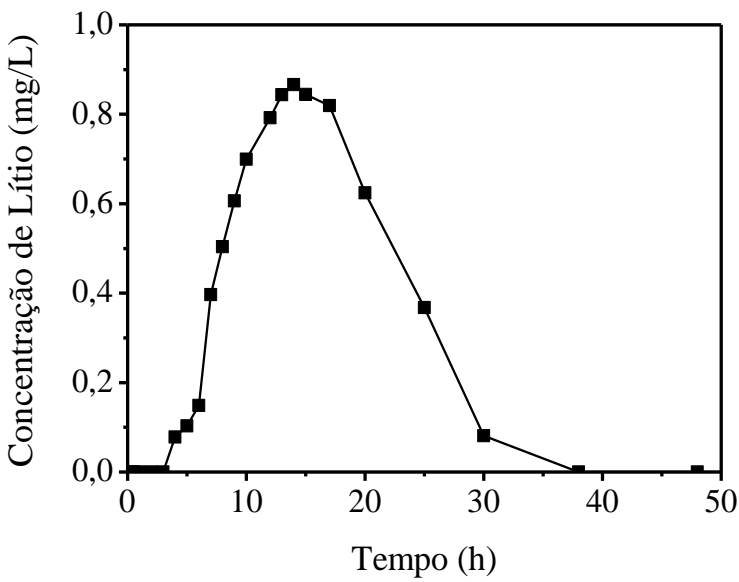

(c)

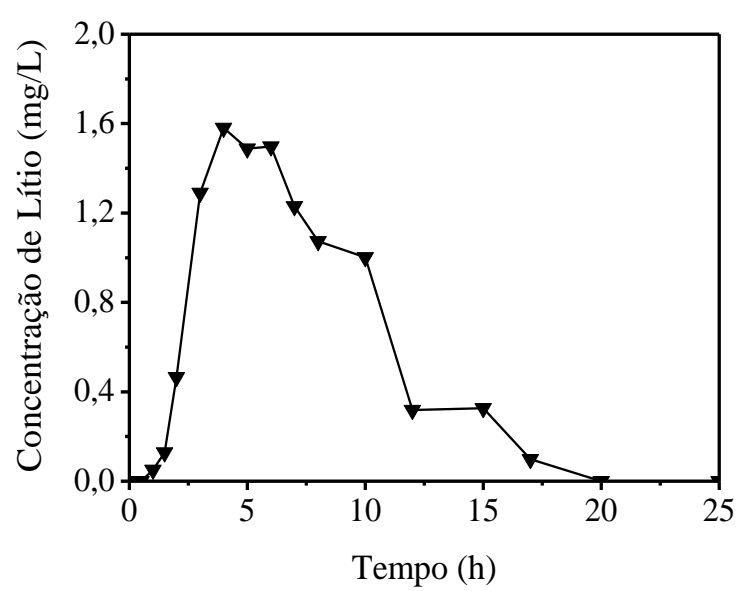

(b)

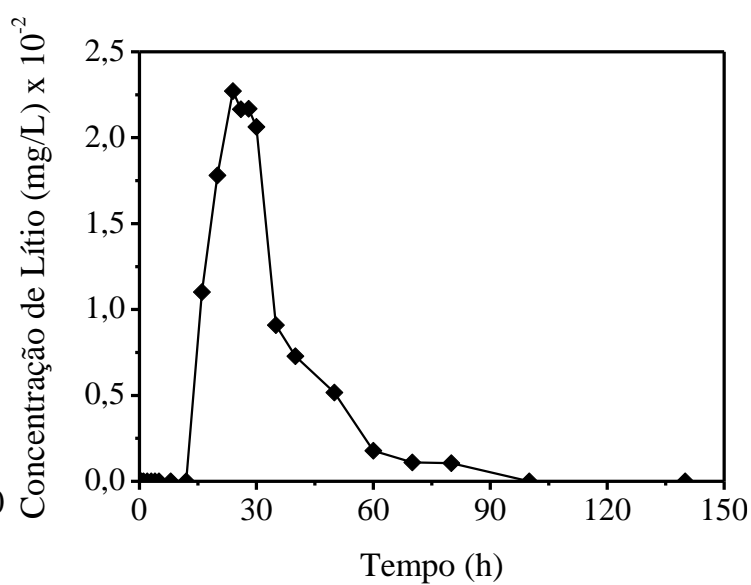

(d)

Figura 2 - Concentração de lítio em função do tempo na saída dos tanques: a) 04 b) 05; c) 06; d) 24 
Os valores da concentração de lítio na solução em função do tempo na saída dos tanques avaliados são apresentados na Figura 2. O decaimento exponencial observado na Figura 2a é típico de um reator bem misturado (análogo a um continuous stirred tank reactor, CSTR) enquanto que os comportamentos apresentados nas Figuras 2b, 2c e 2d são típicos de reatores de fluxo pistonado com presença de dispersão axial (LEVENSPIEL, 1999).

A correlação entre os tempos médios de residência, calculados a partir da Equação (4), e os tempos nominais de residência, calculados através da Equação (9), é apresentada na Figura 3. Uma função potência foi observada, vide Equação (11), com um coeficiente de correlação elevado $\left(R^{2}=\right.$ 0,997). A posição dos pontos experimentais esta abaixo da curva de simetria, ou seja, os tempos médios de residência são menores que os valores nominais, o que pode ser explicado pela redução do volume útil dos tanques em decorrência da presença das partes mecânica usadas para a agitação da polpa, além da presença da fase gasosa e da deposição de sólidos nas paredes dos tanques.

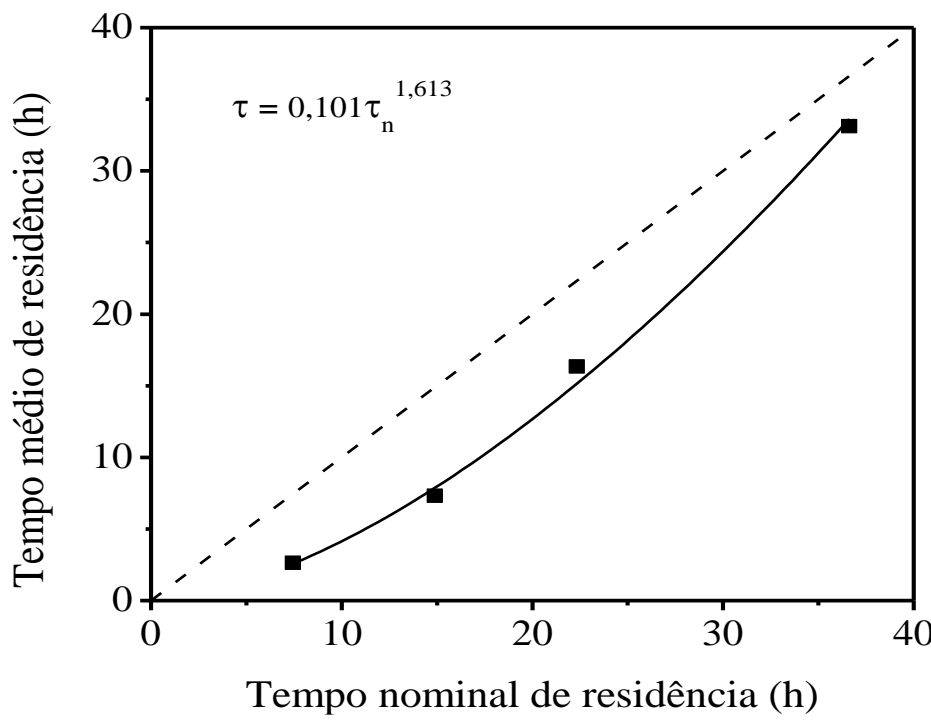

Figura 3 - Correlação entre os tempos médio e nominal de residência

As distribuições do tempo de residência adimensionais, $E(\theta)$, apresentada na Figura 4, indicam que o padrão hidrodinâmico do tanque 04 se assemelha ao de um reator de mistura perfeita (CSTR) modificado por um pequeno deslocamento no início da curva. Já os padrões hidrodinâmicos dos tanques 05 e 06 se assemelham a distribuição de um reator de fluxo pistonado (PFR) com presença de forte dispersão axial. A assimetria observada pode estar associada à presença de zonas de estagnação. As distribuições adimensionais indicam que o aumento do número de tanques na cascata resulta na redução da dispersão e da assimetria da distribuição. Comportamento diferente é observado na distribuição do tanque 24 . O padrão da curva obtida se aproxima de um PFR à medida que a dispersão da curva é reduzida, porém, a assimetria é significativamente aumentada, o que indica a presença de zona de estagnação ao longo da cascata. $\mathrm{O}$ afastamento das tendências apresentados pelas distribuições dos tanques 05 e 06, de redução de dispersão e assimetria, pode esta relacionado com a composição do circuito que é dotado por tanques de dois volumes diferentes. 


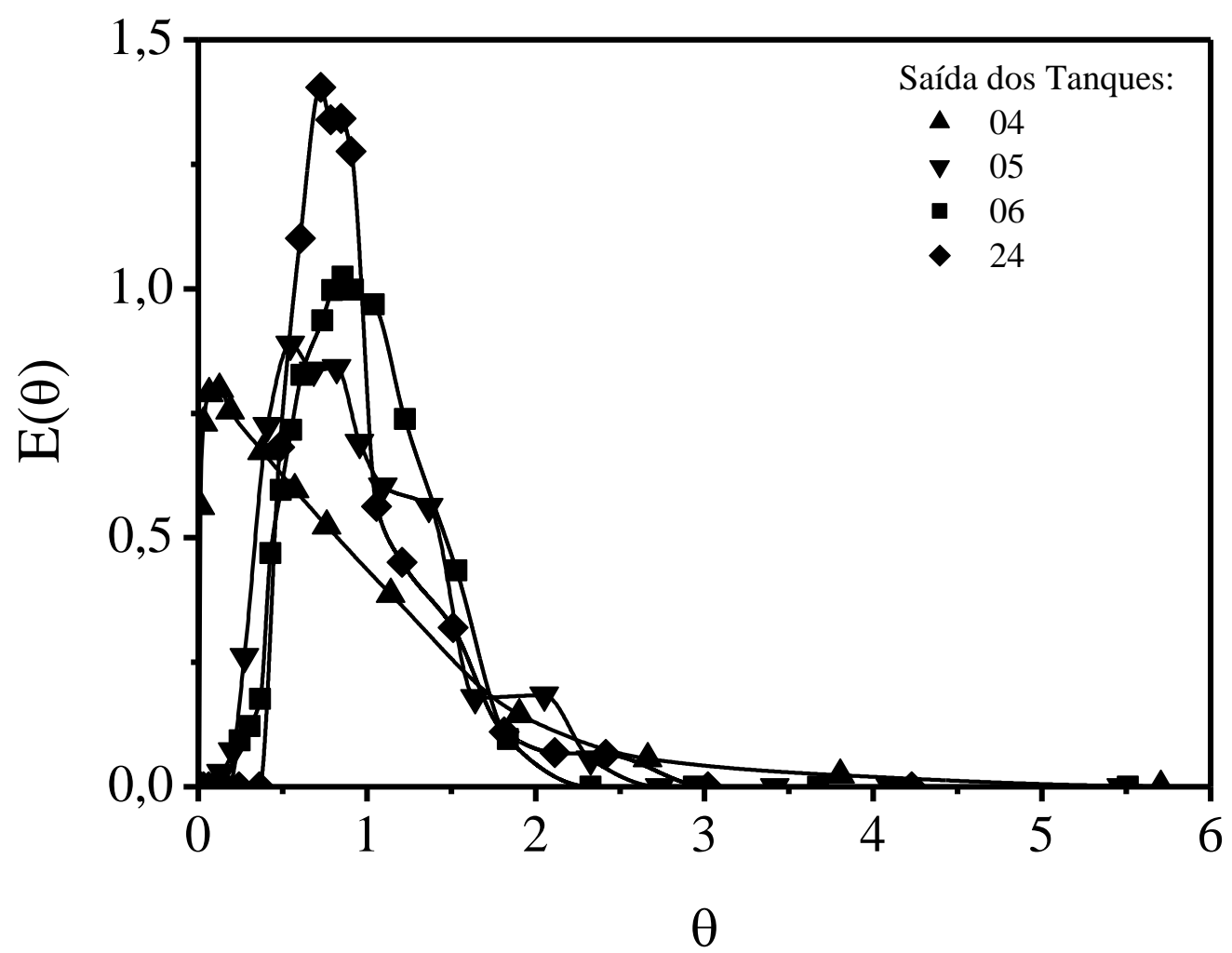

Figura 4 - Distribuição dos tempos de residência adimensional em função do tempo adimensional para a cascata de reatores

Um modelo compartimentado, que se baseia na combinação de reatores ideais simples como CSTR e PRF em diferentes arranjos e configurações, foi utilizado para ajustar as distribuições dos circuitos (BAZIN e HODOUIN, 1988). Para isto, um algoritmo foi criado para ajustar o arranjo de reatores ideais à distribuição experimental $\mathrm{E}(\mathrm{t})$, estimando os parâmetros e simulando a resposta do sistema. Os parâmetros considerados, para o ajuste do modelo aos dados experimentais, foram o tempo médio de residência de cada tanque, a fração do volume do reator ocupado por zonas estagnada além do percentual do fluxo de fluidos trocados entre as zonas ativa e estagnada. Um método de otimização convencional foi usado para minimizar o critério dos mínimos quadrados (DE ANDRADE LIMA e HODOUIN, 2005).

Um grande número de arranjos foi avaliado para representar os diferentes padrões hidrodinâmicos dos circuitos, mas os resultados mostrados são apenas aqueles que melhor ajustaram aos dados experimentais com um número limitado de parâmetros. Todas as distribuições foram ajustadas com um arranjo de reatores ideais em série composto por $\mathrm{K}$ reatores de fluxo pistonado, $L$ reatores de mistura perfeita e $M$ reatores de mistura perfeita com troca entre zona ativa e zona estagnada. A superestrutura genérica que descreve o comportamento hidrodinâmico dos circuitos é apresentada na Figura 5. Nesta figura, $\tau_{1}$, $\tau_{2}$ e $\tau_{3}$ são os tempos médios de residência de cada reator do modelo, $K$, L e $\mathrm{M}$ correspondem ao número de reatores de cada tipo na cascata. Os parâmetros $\lambda$ e $f_{a}$ são à fração do fluxo de fluido trocado entre as zonas ativa e estagnada e a fração do volume do reator ocupado pela zona ativa, respectivamente (DE ANDRADE LIMA e HODOUIN, 2005). 


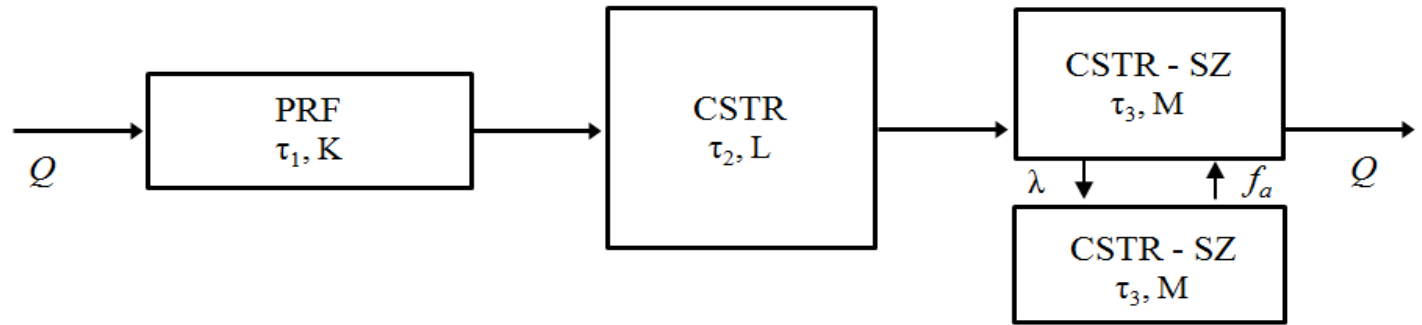

Figura 5 - Modelo hidrodinâmico genérico para o circuito de lixiviação industrial.

A função genérica do sistema, no domínio de Laplace, é dada pela equação:

$F(s)=F_{p}(s)^{K} F_{c}(s)^{L} F_{d}(s)^{M}$

onde $F_{c}(\mathrm{~s})$ representa a função de transferência dos CSTR, $F_{p}(s)$ a função de transferência do PFR e $F_{d}(\mathrm{~s})$ representa a função de transferência do CSTR com troca entre a zona ativa e a zona estagnada (DE ANDRADE LIMA e HODOUIN, 2005).

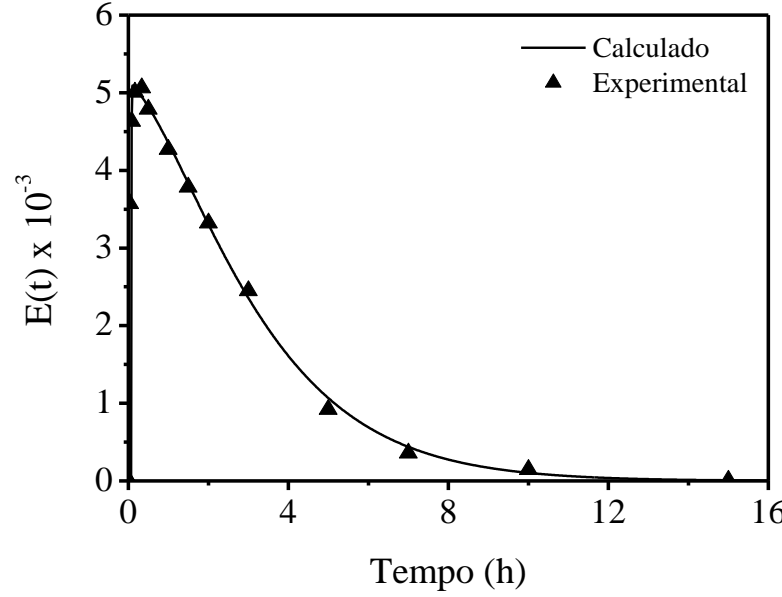

(a)

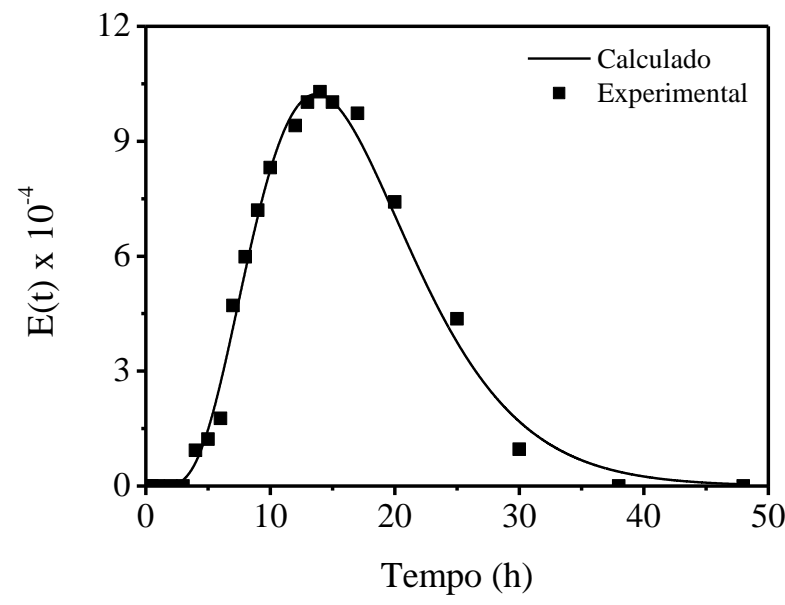

(c)

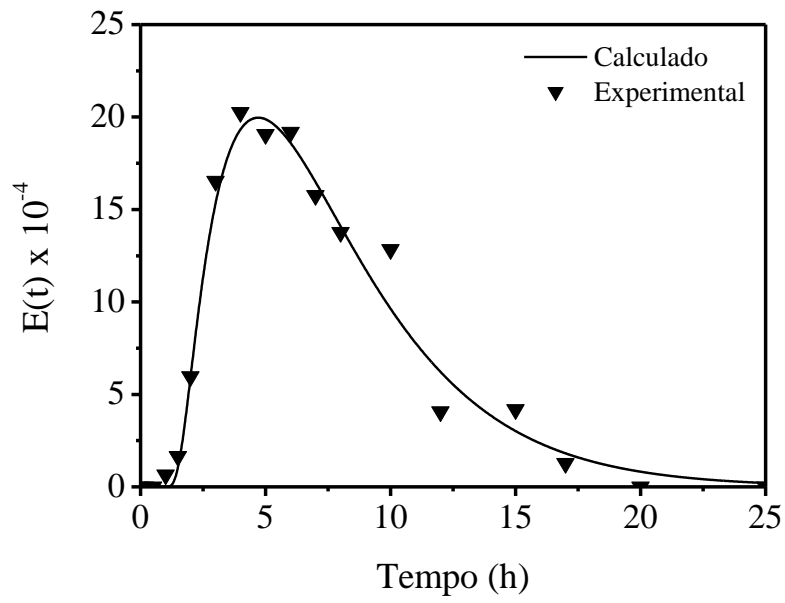

(b)

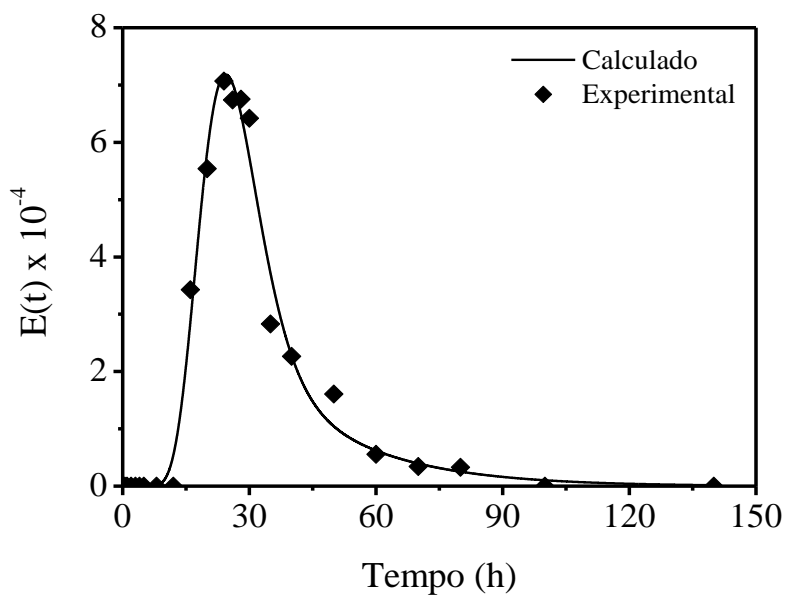

(d)

Figura 6 - Distribuição dos tempos de residência experimental e calculada na saída dos tanques: a) 04; b) 05; c) 06; d) 24

Na Figura 6, são mostradas as distribuições experimentais e calculadas para as saídas dos tanques. A distribuição do tanque 04 , Figura $6 a$, foi ajustada com uma associação em série contendo um PFR, um CSTR e uma cascata com quatro CSTR-SZ pequenos. De maneira análoga, as 
distribuições dos tanques 05, 06 e 24, Figuras 6b, 6c e 6d, respectivamente, foram ajustados modificando apenas o número de reatores CSTR das séries. A saída antecipada de fluidos ou formação de caminhos preferenciais em reatores pode ser identificada com a presença de um pico estreito no início da curva da DTR (BAZIN e HODOUIN, 1988; LEVENSPIEL, 1999). Assim, a falta de ramificações nos modelos, após os ajustes, indica a ausência de formação de caminhos preferenciais.

$\mathrm{Na}$ Tabela 1, é apresentada a correspondência entre os volumes dos reatores ideais do modelo e o volume real dos circuitos. Nesta tabela, $V_{K}$ representa o volume ocupado pelo PFR, $V_{L}$

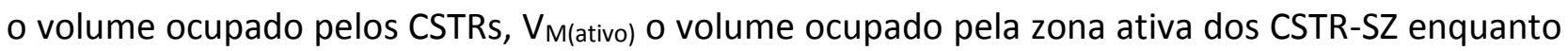

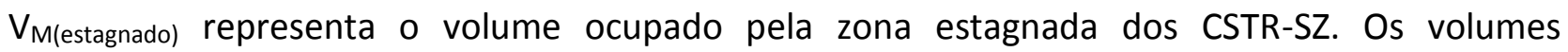
apresentados correspondem à fração do volume total do reator.

Tabela 1 - Distribuição dos volumes dos reatores referente a Figura 6 (\%)

\begin{tabular}{ccccc}
\hline & TQ 04 & TQ 05 & TQ 06 & TQ 24 \\
\hline$V_{K}$ & 2,5 & 12,7 & 3,2 & 7,7 \\
$V_{L}$ & 63,1 & 65,2 & 63,7 & 63,5 \\
$V_{M}$ (ativo) & 0,4 & 9,1 & 23,4 & 6,0 \\
$V_{M}$ (estagnada) & 34,0 & 13,0 & 9,7 & 22,8 \\
\hline
\end{tabular}

A modelagem indica que os circuitos são majoritariamente explicados pela associação de misturadores perfeitos correspondendo a mais de $63 \%$ dos volumes das cascatas, sendo consistente com os tempos médios de residência calculados. Os volumes ocupados por zonas de estagnação não apresentam tendência definida, entretanto avaliando apenas os volumes estagnados dos tanques 04,05 e 06 pode-se observar uma tendência de redução do volume da zona estagnada em detrimento do aumento do volume da zona ativa, à medida que o número de tanques de igual tamanho é aumentado na cascata. O tanque 24 apresenta comportamento diferente. Embora, a distribuição obtida na saída deste tanque possa ser satisfatoriamente explicada através de um arranjo semelhante de reatores ideais, a tendência de redução do volume das zonas de estagnação não é verificada. Este comportamento pode estar associado com a presença de tanques de diferentes volumes na cascata.

\section{CONCLUSÕES}

A distribuição do tempo de residência do circuito industrial de lixiviação de ouro foi obtida a partir do teste com traçador inerte cloreto de lítio. Os resultados mostram que os tempos médios de residência são menores que os tempos nominais o que sugere a redução do volume útil dos tanques. As distribuições adimensionais indicam a presença de atraso na saída dos elementos de fluidos e zonas de estagnação. Na modelagem compartimentada, uma superestrutura genérica, composta por reatores ideais em série, foi identificada após o ajuste das distribuições experimentais. O modelo genérico é composto por PFR, CSTR e CSTR-SZ. A falta de ramificações nos modelos indica a ausência de formação de caminhos preferenciais. A modelagem indica que $63 \%$ do volume nominal dos circuitos são explicados pela associação de CSTRs e que o aumento do número de tanques de iguais volumes na cascata implica na redução do volume das zonas de estagnação. O modelo encontrado pode ser usado em associação com as equações cinéticas de dissolução de ouro para simular mais realisticamente os processos de lixiviação industrial. 


\section{AGRADECIMENTOS}

Os autores agradecem a Mineração Fazenda Brasileiro pelo apoio para a realização da parte experimental deste trabalho e ao Professor Sergio Luis Costa Ferreira (Departamento de Química Analítica da UFBA), pela realização das análises químicas do traçador.

\section{REFERÊNCIAS}

1. BAZIN, C., HODOUIN, D. Residence time distributions modeling for mineral processes evaluation. CIM Bull, v.81, n.911, p. 115-124, 1988.

2. DANCKWERTS, P. V. Continuous flow systems. Distribution of residence times. Chemical Engineering Science, v. 2, p. 1-13, 1953.

3. DE ANDRADE LIMA, L.R.P., HODOUIN, D. Residence time distribution of an industrial mechanically agitated cyanidation tank. Minerals Engineering, v.18, n. 6, p. 613-621, 2005.

4. HABASHI, F. One Hundred Years of Cyanidation. Canadian Institute of Mining, Metallurgy and Petroleum, v. 80, n. 905, p. 108-114, 1987.

5. HIMMELBLAU, D.M., BISCHOFF, K.B. Process analysis and simulation: deterministic systems. John Wiley \& Sons, New York, 1968.

6. LELINSKI, D., ALLEN, J., REDDEN, L., WEBER, A. Analysis of the residence time distribution in large flotation machines. Minerals Engineering, v.15, p. 499-505, 2002.

7. LEVENSPIEL, O. Chemical reaction engineering, 3rd ed., John Wiley \& Sons, New York, 1999.

8. NAUMAN, E.B., BUfFHAM, B.A. Mixing in continuous flow systems, John Wiley \& Sons. New York, 1983.

9. NEWCOMBE, B., BRADSHAW, D., WIGHTMAN, E. The hydrodynamics of an operating flash flotation cell. Minerals Engineering, v.41, p. 86-96, 2013.

10. STEGOWSKI, Z., DAGADU, C. P. K., FURMAN, L., AKAHO, E. H. K., DANSO, K. A., MUMUNI, I. I., ADU, P. S., AMOAH, C. Determination of flow patterns in industrial gold leaching tank by radiotracer residence time distribution measurement. Nukleonika, v.55, n.3, p. 339-344, 2010. 